REZENSIONEN

\section{Transdisziplinäre Forschung: Let's do it!}

\section{Bergmann et al. / ISOE: Qualitätskrite- rien transdisziplinärer Forschung. Ein Leitfaden für die formative Evaluation von Forschungsprojekten. Frankfurt a. M., 2005, (ISOE-Studientexte Nr. 13); 76 S., ISSN 0947-6083, Euro 8,00 ${ }^{1}$}

\section{Chr. Pohl, G. Hirsch Hadorn: Gestaltungs- prinzipien für die transdisziplinäre For- schung. Ein Beitrag des td-net. München, 2006, oekom Verlag, 120 S., ISBN 10: 3- 86581-000-4, Euro 29,80²}

\section{Rezension von Michael Decker, ITAS}

\section{Hintergrund}

Der Erarbeitung von adäquaten Lösungsvorschlägen für gesellschaftliche und politische Probleme sowie für Probleme aus der Lebenswelt erfordert veränderte Formen der Wissensproduktion: Wissenschaft muss für diesen Zweck ,post-normal“" werden oder in einen anderen Modus, nämlich den „Mode 2“ übergehen. ${ }^{3}$ Über die Anforderungen, die daraus für die Wissensproduktion erwachsen, herrscht eigentlich Einigkeit:

- Die konkrete gesellschaftliche Situation stellt einen starken Kontextbezug dar; gefordert ist also Wissen für genau diese Entscheidungs-/Handlungssituation.

- Lebensweltliche Probleme sind nicht nach wissenschaftlichen Disziplinen ,geordnet“ eine inter- bzw. transdisziplinäre Herangehensweise ist erforderlich.

- Durch den Kontextbezug und die disziplinübergreifende Forschung kommen zusätzliche wissenschaftliche, aber auch gesellschaftliche und politische Qualitätskriterien ins Spiel - die Qualität des Lösungsvorschlags für ein lebensweltliches Problem muss sich in der Lebenswelt messen lassen.

- Die Wissensproduktion wird gesellschaftlich rechtfertigungspflichtig und reflexiv. Forschung muss sich verstärkt an sozialen
Werten und politischen Zielen orientieren und sich über diese legitimieren.

Formuliert man diese Anforderungen in einem Satz, so könnte dieser lauten: Um für die Lösung eines lebensweltlichen Problems qualitativ hochwertiges Wissen zu produzieren, das sich an gesellschaftlichen Werten orientiert, muss transdisziplinär geforscht werden. Die Rahmenkonzepte für diese Art Wissensproduktion - außer den hier genannten des „Mode 2“ und der ,postnormal science“ gibt es noch weitere - halten sich zurück, wenn es um die konkrete methodische Ausgestaltung von transdisziplinären Projekten geht.

Dieser Aufgabe haben sich nun zum einen das „Evaluationsnetzwerk für transdisziplinäre Forschung" (evalunet) ${ }^{4}$, in dessen Zusammenhang die Publikation von Matthias Bergmann et al. steht - und zum anderen das transdisciplinarity net, td-net ${ }^{5}$, mit der Publikation von Christian Pohl und Gertrude Hirsch Hadorn angenommen. Im Folgenden wird Bergmann et al. 2005 mit „evalunet“ und Pohl / Hirsch Hadorn mit „tdnet" abgekürzt. Sie werden hier gemeinsam behandelt, weil sie sich der Thematik von zwei unterschiedlichen Ausgangspunkten nähern: Die „Gestaltungsprinzipien für die transdisziplinäre Forschung" (td-net) legen den Schwerpunkt auf konzeptionelle Herausforderungen der Transdisziplinären Forschung (TF) und wählen somit die „Vorwärtsrichtung“: Welche Ziele strebt die TF an, unter welchen Randbedingungen findet sie statt, was bedeuten diese Aspekte für die Formulierung und Bearbeitung der Forschungsfragen und in Bezug auf die "in Wert-Setzung“ der Ergebnisse (td-net, S. 12-13). Die „Qualitätskriterien transdisziplinärer Forschung " möchten Wissenschaftlerinnen und Wissenschaftlern, die transdisziplinär forschen, Kriterien an die Hand geben, mit denen sie ihre Arbeit intern evaluieren und somit auch extern eine Qualitätssicherung ihrer Forschungsarbeit erreichen können (evalunet, S. 21). Dabei werden nicht nur Qualitätskriterien beschrieben, sondern auch Aussagen über methodische und Verfahrensfragen gemacht (evalunet, S. 7). Darüber hinaus bietet sich hier die gemeinsame Vorstellung an, weil die dahinter stehenden Akteursgruppen durchaus miteinander verzahnt sind, wie ein Blick in die angegebenen Listen der Kommentatoren und externen Experten verrät. 


\section{Gestaltungsprinzipien der trans- disziplinären Forschung (td-net)}

Das Autorenteam Christian Pohl und Gertrude Hirsch Hadorn gliedert den TF-Prozess in drei Phasen: 1. die Problemidentifikation, 2. die Problembearbeitung und 3. die In-Wert-Setzung. Als generelles Gestaltungsprinzip wird diesem Tripel das „rekursive Vorgehen“ übergeordnet, welches sowohl für den gesamten TF-Prozess als auch für jede der drei Phasen gilt. Beispielsweise ermöglicht das rekursive Vorgehen, dass in der Phase der Problembearbeitung erarbeitete Erkenntnisse zu einer Korrektur der ursprünglichen Problemidentifikation führen können.

Mit „Problemen“ sind gesellschaftlich relevante Problemfelder gemeint, denen sich die $\mathrm{TF}$ in der Art widmet, dass sie die Komplexität der Probleme erfasst und die Diversität der wissenschaftlichen und gesellschaftlichen Sichtweisen der Probleme berücksichtigt. Für die Entwicklung von am Gemeinwohl orientierten, praktischen Lösungsansätzen verbindet sie abstrahierende Wissenschaft und fallsspezifisch relevantes Wissen. Diese hehren Ziele der TF erscheinen den Autoren in konkreten Problemzusammenhängen nur schwer einlösbar (tdnet, S. 18), sodass sie empfehlen, die Ansprüche an den TF-Prozess durch vier Prinzipien, die für alle drei Phasen der TF relevant sind, auf ein einlösbares $\mathrm{Ma} ß \mathrm{zu}$ reduzieren:

1. Durch die Reduktion der Komplexität des Problemfeldes (Spezifizierung des Wissensbedarfs, Formulierung der Forschungsfragen des Projekts) werden entscheidende Faktoren des Projekts festgelegt: Welches Systemverständnis liegt zu Grunde, welche Zielsetzungen werden vereinbart und welche möglichen gesellschaftlichen Transformationsprozesse möchte man in den Blick nehmen. Darüber hinaus werden relevante gesellschaftliche Akteure und wissenschaftliche Disziplinen identifiziert, die dann in den TF-Prozess so eingebunden werden, dass der Diversität der relevanten Sichtweisen Rechnung getragen wird (td-net, S. 17).

2. Die Chance, wirksame Problemlösungen für die Lebenswelt zu erarbeiten, wird durch die wirkungsbezogene Einbettung des Projekts in seine Umwelt erreicht. Dabei sind der Stand der Wissenschaft auf die identifi- zierten Probleme und der Stand der lebensweltlichen Problemlage zu berücksichtigen.

3. Die Integrationsleistung, die ein TF-Prozess für die Erarbeitung von Problemlösungen erbringen muss, wird, so Pohl und Hirsch Hadorn, durch ein „offenes Aufeinanderzugehen" erreicht, das sich im Wesentlichen dadurch auszeichnet, dass alle Teilnehmenden bereit sind, die eigene Sichtweise zu den jeweils anderen in Relation $\mathrm{zu}$ stellen und damit als potenziell relevant zu akzeptieren.

4. Durch eine rekursive Gestaltung des TFProzesses wird die Möglichkeit eröffnet, einzelne Projektschritte mehrfach zu durchlaufen, um einen gezielten Lerneffekt zu erreichen. Diese (Zwischen-)Ergebnisse der In-Wert-Setzung dienen dann als Grundlage für eine Neuausrichtung der Problemidentifikation und -bearbeitung, die wiederum in eine In-Wert-Setzung mündet.

Diese vier Prinzipien und die drei Phasen des transdisziplinären Forschungsprozesses werden im Detail erläutert und durch viele, anschaulich gewählte Beispiele illustriert. Fünf in Matrixform dargestellte "Arbeitsinstrumente“ sollen bei der konkreten Gestaltung von TF-Projekten helfen. Sie fokussieren auf bereits erwähnte Kernaspekte: Verortung der Beteiligten (1) und des Wissensbedarfs (2), Form der Zusammenarbeit und Integration (3) und auf die Einbettung in die Lebenswelt (4) und das wissenschaftliche Umfeld (5). Sie befinden sich jeweils am Ende der ausführlichen Darstellung des Themas und werden nochmals extra mit normativ formulierten Hinweisen zur konkreten Gestaltung erläutert.

\section{Qualitätskriterien trandisziplinärer Forschung (evalunet)}

Der Untertitel „Ein Leitfaden für die formative Evaluation von (transdisziplinären) Forschungsprojekten“ ist hier Programm. Das siebenköpfige Autorenteam ${ }^{6}$ hält sich nicht mit langen Vorreden auf und liefert nach einer kurzen Einleitung - in der auch auf das methodische Vorgehen zur Gewinnung der Qualitätskriterien eingegangen wird - bereits die erste Kurzanleitung, die in sechs Schritten eine formative Projektevaluation ermöglichen soll.

Die Kennzeichen der TF werden dabei ganz ähnlich wie in den „Gestaltungsprinzi- 
pien" formuliert. Die Orientierung an konkreten Problemen der Lebenswelt, für die Lösungsansätze erarbeitet werden sollen, steht im Zentrum. Dafür ist zunächst eine gemeinsame Übersetzung des Problems in Forschungsfragen nötig, für deren Beantwortung dann sowohl wissenschaftliches als auch Praxiswissen herangezogen werden. Methodischer Kern der TF ist die Integration der unterschiedlichen disziplinären und praktischen Wissensbestände, die schließlich in konkrete Handlungsempfehlungen münden sollen. Um die Adäquatheit dieser Handlungen darlegen zu können, müssen verschiedene Wirkebenen Berücksichtigung finden. Im Prozess der TF werden dann drei Projektphasen unterschieden:

1. Formulierung des gemeinsamen Forschungsgegenstands und Teambildung: Ausgehend vom Problem wird in dieser Phase das Projektteam (aus Wissenschaft und Praxis) gebildet, welches dann die Formulierung der im Projekt zu bearbeitenden Forschungsfragen übernimmt.

2. Projektdurchführung und Methodik: Die Forschungsfragen werden in bearbeitbaren Teilfragen beantwortet (Differenzierung) und anschließend wieder entsprechend integriert. Wissensgenerierung und die Erarbeitung von Anschlussfähigkeiten zwischen den Teilprojekten ist hier zentral.

3. Transdisziplinäre Integration: Schließlich werden die Ergebnisse der Module in Hinblick auf die Problemstellung integriert und daraus Impulse für Handlungsstrategien im konkreten Handlungskontext entwickelt.

Entlang dieser Projektphasen entwickelt das Autorenteam sehr detaillierte Qualitätskriterien, anhand derer die für die einzelnen Phasen entscheidenden Aspekte beurteilt werden können. Die Qualitätskriterien sind jeweils als Frage formuliert, für deren Beantwortung Alternativen angegeben werden. So entsteht nicht nur ein Leitfaden, sondern sogar eine Checkliste, die man für die interne oder externe Evaluierung von TF-Projekten anwenden kann.

Die Autoren formulieren neben den so genannten Basis-Kriterien auch noch Detailkriterien, wobei genau genommen die Basiskriterien aus den Detailkriterien ,,herausdestilliert" sind. Mit dem Hinweis, dass der Aufwand für die Evaluation in einem vernünftigen
Verhältnis zu Projektgröße stehen soll, wird empfohlen, sich aus beiden Kriteriensets eine auf das jeweilige Projektsetting ,passende“ Checkliste zusammenzustellen (evalunet, S. 22). Dabei ist es sicher sinnvoll, auch für die Basiskriterien die nur in der der Rubrik „Detailkriterien" formulierten Anforderungen zur Erläuterung nochmals nachzulesen.

\section{Diskussion}

Beide Autorenteams fokussieren auf die Kernelemente der transdisziplinären Forschung. Der Problembezug wird nicht nur deutlich herausgestellt und, verbunden damit, eine klare Formulierung der aus der Problemstellung resultierenden Forschungsfragen gefordert. Es wird auch in beiden Texten darüber hinaus darauf hingewiesen, dass in diesem frühen Schritt schon viel über den Erfolg oder den Misserfolg des TFProjekts entschieden wird. Evalunet hebt in diesem Zusammenhang hervor, dass man der Weichenstellung in dieser frühen Phase besonderes Augenmerk schenken muss, gerade wenn man die Evaluation auch als einen Lernprozess in dem Projekt verstehen will (evalunet, S. 23). Analog unterstreicht td-net, dass in diesem Schritt, der als Kernstück der Forschung bezeichnet wird, grundlegende Vorentscheidungen getroffen werden. Dieser Hinweis ist zwar durch die Möglichkeit des rekursiven Durchlaufens der einzelnen Projektphasen etwas abgeschwächt (man kann die Forschungsfrage ja reformulieren), realistischer Weise muss man hier aber davon ausgehen, dass nur moderate Änderungen, z. B. auch in der Zusammenstellung der Akteure, vorgenommen werden können, wenn der gesamte Projektablaufplan nicht völlig aus den Fugen geraten soll.

Von kleinen Aspekten abgesehen - beispielsweise wird in den Anforderungen zum Qualitätskriterium „A2.1 Greift das Projekt eine lebensweltliche Problemstellung auf und worin besteht ihre Relevanz" explizit darauf hingewiesen, dass man auf einen Bezug auf das „Gemeinwohl“" verzichtet, wenn man die akteursbezogene Relevanz beschreibt (evalunet, S. 42), während td-net (S. 16) ganz zentral bemerkt, dass $\mathrm{TF}$ „Wissen zu einer am Gemeinwohl orientieren praktischen Lösung von Problemen" liefert - passen die in beiden Büchern verwendete Strukturierung der TF-Prozesses sehr gut zusammen. Besonders offen- 
sichtlich wird das bei der Beschreibung der starken Rolle der „In-Wert-Setzung“. Gleichzeitig ergänzen sich die Texte sehr gut zu einem Gesamtbild der TF, weil die strenge Formulierung der Detailkriterien seitens der Evalunet-Autoren einige vom td-net entwickelte Gestaltungsprinzipien (die verbunden mit der Rolle des Gestalters ja immer auch noch verhandelbare Aspekte beinhalten) aus der Sicht des Evaluators auf den Punkt bringt.

Diese Einigkeit findet man auch in einer Unterlassung wieder! Beide Texte gehen erstaunlich wenig auf die Entwicklung einer gemeinsamen Sprache ein und die damit verbundene Problematik in einem TF-Prozess. Jäger und Scheringer identifizieren dieses Sprachproblem: „... nämlich dass Begriffe und Theorien, die mit eingesetzten Methoden verbunden sind, außerhalb ihres ursprünglichen Kontexts nicht mehr klar definiert und verständlich sind. Neben den Methoden müssen darum auch die Begriffe angepasst und zum Teil neu entwickelt werden" (Jäger, Scheringer 1998, S. 16 f.). Ähnlich äußern sich auch Schmidt und Grunwald (2005). Sowohl Evalunet als auch td-net gehen auf diesen Aspekt nur über die „Integration“ ein. In einer Auflistung der Integrationsarten (td-net, S. 54 f.) wird an drei Stellen auf die integrative Funktion von Begriffsbestimmungen/-übertragungen eingegangen. Bei Evalunet ist es am ehesten das Detailkriterium 6, wo in den Anforderungen die gemeinsame Sprache erwähnt wird.

Diese Ähnlichkeit in der Argumentation verbunden mit den unterschiedlichen Blickwinkeln - legt die Empfehlung zur Lektüre beider Bücher nahe. Das sei hiermit für Koordinatorinnen und Koordinatoren von transdisziplinären Forschungsprojekten auch ausdrücklich empfohlen. Wobei man in Anbetracht der moderaten Preise beider Texte dem Rezensent diese Entscheidungsschwäche nachsehen möge. Die Komplementarität führt sich auch im Anhang fort. Pohl und Hirsch Hadorn präsentieren dort eine wirklich beeindruckende Sammlung und Klassifizierung von sage und schreibe 21 Definitionen zur TF. Bergmann et al. ergänzen ihren Kriterienkatalog um eine Matrix, in der dieselben Kriterien in einem themenzentrierten Evaluationsschema verortet wird. Eine Ergänzung, die sicherlich in konkreten Evaluationszusammenhängen Verwendung finden wird.

\section{Anmerkungen}

1) Bergmann et al. 2005 ist zu beziehen über das Institut; auch als kostenpflichtiger download im Internet verfügbar unter http://downloads.isoe.de.

2) Die englische Fassung „Beyond Disciplinary Boundaries" ist ab März 2007 unter der ISBN 13: 978-3-86581-046-5 erhältlich.

3) Für den „Post-normal Science“-Ansatz siehe Funtowicz/Ravetz 1993 und Ravetz/Funtowicz 1999; für den Ansatz der „Knowledge production in Mode 2" siehe Gibbons et al. 1994, Nowotny et al. 2001.

4) Bei Evalunet handelt es sich um ein fünfjähriges Projektvorhaben von fünf Instituten des Ökoforums unter Federführung des ISOE und der Leitung von Matthias Bergmann vom Wissenschaftskolleg zu Berlin; es wurde im April 2006 abgeschlossen (Anm. d. Red.).

5) td-net steht für ,network for transdisciplinarity in sciences and humanities", wurde im Januar 2003 von der Swiss Academy of Sciences / Akademie für Naturwissenschaften initiiert; es verfügt über das Internet Portal http://www.transdisciplinarity. ch/ueber_tdnet.html (Anm. d. Red.).

6) Die Mitglieder des Autorenteam sind neben Mattias Bergmann: Bettina Brohmann, Esther Hofmann, M. Céline Loibl, Regine Rehaag, Engelbert Schramm, Jan-Peter Voß.

\section{Literatur}

Funtowicz, S.; Ravetz J.R., 1993: Science for the post-normal age. In: FUTURES 25 (1993), S. 739755

Funtowicz, S.; Ravetz, J.R., 1999: Post-Normal Science - An Insight Now Maturing. In: FUTURES 31 (1999), S. 641-646

Gibbons, M.; Limoges, C.; Nowotny, H. et al., 1994: The new production of knowledge: Dynamics of Science and Research in Contemporary Societies. London: SAGE

Jaeger, J.; Scheringer, M., 1998: Transdisziplinarität. Problemorientierung ohne Methodenzwang. In: GAIA, 7/1 (1998), S. 10-25

Nowotny, H.; Scott, P.; Gibbons, M., 2001: ReThinking Science. Knowledge and the Public in an Age of Uncertainty. Cambridge: Polity

Schmidt, J.C.; Grunwald, A., 2005: Einführung in den Schwerpunkt: Methodische Fragen der Interund Transdisziplinarität. In: Technikfolgenabschätzung Theorie und Praxis 14/2 (2005), S. 4-11 